\title{
Set-Valued Additive Functional Equations
}

\author{
ChOOnkil PARK, SungsiK Yun*, Jung Rye LeE, And DONG Yun SHIN
}

ABSTRACT. In this paper, we introduce set-valued additive functional equations and prove the Hyers-Ulam stability of the set-valued additive functional equations by using the fixed point method.

Keywords: Hyers-Ulam stability; set-valued additive functional equation; fixed point.

2010 Mathematics Subject Classification: 47H10, 54C60, 39B52, 47H04.

\section{INTRODUCTION AND PRELIMINARIES}

Set-valued functions in Banach spaces have been developed in the last decades. The pioneering papers by Aumann [4] and Debreu [11] were inspired by problems arising in Control Theory and Mathematical Economics. We can refer to the papers by Arrow and Debreu [2], McKenzie [24], the monographs by Hindenbrand [18], Aubin and Frankowska [3], Castaing and Valadier [7], Klein and Thompson [22] and the survey by Hess [17].

The stability problem of functional equations originated from a question of Ulam [37] concerning the stability of group homomorphisms. Hyers [19] gave a first affirmative partial answer to the question of Ulam for Banach spaces. Hyers' Theorem was generalized by Aoki [1] for additive mappings and by Rassias [35] for linear mappings by considering an unbounded Cauchy difference. A generalization of the Rassias theorem was obtained by Găvruta [16] by replacing the unbounded Cauchy difference by a general control function in the spirit of Rassias' approach

The functional equation $f(x+y)=f(x)+f(y)$ is called an additive functional equation. In particular, every solution of the additive functional equation is said to be an additive mapping. The functional equation $2 f\left(\frac{x+y}{2}\right)=f(x)+f(y)$ is called a Jensen additive functional equation. In particular, every solution of the Jensen additive functional equation is said to be a Jensen additive mapping. The stability problems of several functional equations have been extensively investigated by a number of authors and there are many interesting results concerning this problem (see $[15,16,20,36])$.

Let $(X, d)$ be a generalized metric space. An operator $T: X \rightarrow X$ satisfies a Lipschitz condition with Lipschitz constant $L$ if there exists a constant $L \geq 0$ such that $d(T x, T y) \leq$ $L d(x, y)$ for all $x, y \in X$. If the Lipschitz constant $L$ is less than 1 , then the operator $T$ is called a strictly contractive operator. Note that the distinction between the generalized metric and the usual metric is that the range of the former is permitted to include the infinity. We recall the following theorem by Margolis and Diaz.

Theorem 1.1. [8, 12] Let $(X, d)$ be a complete generalized metric space and let $J: X \rightarrow X$ be a strictly contractive mapping with Lipschitz constant $L<1$. Then for each given element $x \in X$, either

$$
d\left(J^{n} x, J^{n+1} x\right)=\infty
$$

Received: 27 February 2019; Accepted: 8 April 2019; Published Online: 9 April 2019

*Corresponding author: Sungsik Yun; ssyun@hs.ac.kr

DOI: $10.33205 / \mathrm{cma} .528182$ 
for all nonnegative integers $n$ or there exists a positive integer $n_{0}$ such that

(1) $d\left(J^{n} x, J^{n+1} x\right)<\infty, \quad \forall n \geq n_{0}$;

(2) the sequence $\left\{J^{n} x\right\}$ converges to a fixed point $y^{*}$ of $J$;

(3) $y^{*}$ is the unique fixed point of $J$ in the set $Y=\left\{y \in X \mid d\left(J^{n_{0}} x, y\right)<\infty\right\}$;

(4) $d\left(y, y^{*}\right) \leq \frac{1}{1-L} d(y, J y)$ for all $y \in Y$.

In 1996, Isac and Rassias [21] were the first to provide applications of stability theory of functional equations for the proof of new fixed point theorems with applications. By using fixed point methods, the stability problems of several functional equations have been extensively investigated by a number of authors (see $[9,10,13,26,32,34])$.

Let $Y$ be a Banach space. We define the following:

$2^{Y}$ : the set of all subsets of $Y$;

$C_{b}(Y)$ : the set of all closed bounded subsets of $Y$;

$C_{c}(Y)$ : the set of all closed convex subsets of $Y$;

$C_{c b}(Y)$ : the set of all closed convex bounded subsets of $Y$.

On $2^{Y}$ we consider the addition and the scalar multiplication as follows:

$$
C+C^{\prime}=\left\{x+x^{\prime}: x \in C, x^{\prime} \in C^{\prime}\right\}, \quad \lambda C=\{\lambda x: x \in C\},
$$

where $C, C^{\prime} \in 2^{Y}$ and $\lambda \in \mathbb{R}$. Further, if $C, C^{\prime} \in C_{c}(Y)$, then we denote by $C \oplus C^{\prime}=\overline{C+C^{\prime}}$.

It is easy to check that

$$
\lambda C+\lambda C^{\prime}=\lambda\left(C+C^{\prime}\right), \quad(\lambda+\mu) C \subseteq \lambda C+\mu C .
$$

Furthermore, when $C$ is convex, we obtain $(\lambda+\mu) C=\lambda C+\mu C$ for all $\lambda, \mu \in \mathbb{R}^{+}$.

For a given set $C \in 2^{Y}$, the distance function $d(\cdot, C)$ and the support function $s(\cdot, C)$ are respectively defined by

$$
\begin{aligned}
d(x, C) & =\inf \{\|x-y\|: y \in C\}, \quad x \in Y, \\
s\left(x^{*}, C\right) & =\sup \left\{\left\langle x^{*}, x\right\rangle: x \in C\right\}, \quad x^{*} \in Y^{*} .
\end{aligned}
$$

For every pair $C, C^{\prime} \in C_{b}(Y)$, we define the Hausdorff distance between $C$ and $C^{\prime}$ by

$$
H\left(C, C^{\prime}\right)=\inf \left\{\lambda>0: C \subseteq C^{\prime}+\lambda B_{Y}, \quad C^{\prime} \subseteq C+\lambda B_{Y}\right\},
$$

where $B_{Y}$ is the closed unit ball in $Y$.

The following proposition reveals some properties of the Hausdorff distance.

Proposition 1.1. For every $C, C^{\prime}, K, K^{\prime} \in C_{c b}(Y)$ and $\lambda>0$, the following properties hold

(a) $H\left(C \oplus C^{\prime}, K \oplus K^{\prime}\right) \leq H(C, K)+H\left(C^{\prime}, K^{\prime}\right)$;

(b) $H(\lambda C, \lambda K)=\lambda H(C, K)$.

Let $\left(C_{c b}(Y), \oplus, H\right)$ be endowed with the Hausdorff distance $h$. Since $Y$ is a Banach space, $\left(C_{c b}(Y), \oplus, H\right)$ is a complete metric semigroup (see [7]). Debreu [11] proved that $\left(C_{c b}(Y), \oplus, H\right)$ is isometrically embedded in a Banach space as follows.

Lemma 1.1. [11] Let $C\left(B_{Y^{*}}\right)$ be the Banach space of continuous real-valued functions on $B_{Y^{*}}$ endowed with the uniform norm $\|\cdot\|_{u}$. Then the mapping $j:\left(C_{c b}(Y), \oplus, H\right) \rightarrow C\left(B_{Y^{*}}\right)$, given by $j(A)=$ $s(\cdot, A)$, satisfies the following properties:

(a) $j(A \oplus B)=j(A)+j(B)$;

(b) $j(\lambda A)=\lambda j(A)$;

(c) $H(A, B)=\|j(A)-j(B)\|_{u}$;

(d) $j\left(C_{c b}(Y)\right)$ is closed in $C\left(B_{Y^{*}}\right)$

for all $A, B \in C_{c b}(Y)$ and all $\lambda \geq 0$. 
Let $f: \Omega \rightarrow\left(C_{c b}(Y), H\right)$ be a set-valued function from a complete finite measure space $(\Omega, \Sigma, \nu)$ into $C_{c b}(Y)$. Then $f$ is Debreu integrable if the composition $j \circ f$ is Bochner integrable (see [6]). In this case, the Debreu integral of $f$ in $\Omega$ is the unique element $(D) \int_{\Omega} f d \nu \in C_{c b}(Y)$ such tha $j\left((D) \int_{\Omega} f d \nu\right)$ is the Bochner integral of $j \circ f$. The set of Debreu integrable functions from $\Omega$ to $C_{c b}(Y)$ will be denoted by $D\left(\Omega, C_{c b}(Y)\right)$. Furthermore, on $D\left(\Omega, C_{c b}(Y)\right)$, we define $(f+g)(\omega)=f(\omega) \oplus g(\omega)$ for all $f, g \in D\left(\Omega, C_{c b}(Y)\right)$. Then we obtain that $\left(\left(\Omega, C_{c b}(Y)\right),+\right)$ is an abelian semigroup.

Set-valued functional equations have been extensively investigated by a number of authors and there are many interesting results concerning this problem (see [5, 27, 28, 29, 30, 31, 33]).

Using the fixed point method, we prove the Hyers-Ulam stability of the following set-valued additive functional equations

$$
H(F(x+y), F(x) \oplus F(y)) \leq \varphi(x, y)
$$

and

$$
H\left(2 F\left(\frac{x+y}{2}\right), F(x) \oplus F(y)\right) \leq \varphi(x, y) .
$$

Throughout this paper, let $X$ be a real normed space and $Y$ a real Banach space.

\section{StABILITY OF THE SET-VALUED ADDITIVE FUNCTIONAL EQUATION (1.1)}

Using the fixed point method, we prove the Hyers-Ulam stability of the set-valued additive functional equation (1.1).

Definition 2.1. [23] Let $F: X \rightarrow C_{c b}(Y)$. The set-valued additive functional equation is defined by

$$
F(x+y)=F(x) \oplus F(y)
$$

for all $x, y \in X$. Every solution of the set-valued additive functional equation is called a set-valued additive mapping.

Definition 2.2. Let $F: X \rightarrow C_{c b}(Y)$. The set-valued Jensen additive functional equation is defined by

$$
2 F\left(\frac{x+y}{2}\right)=F(x) \oplus F(y)
$$

for all $x, y \in X$. Every solution of the set-valued Jensen additive functional equation is called a setvalued Jensen additive mapping.

Theorem 2.2. Let $\varphi: X^{2} \rightarrow[0, \infty)$ be a function such that there exists an $L<1$ with

$$
\varphi(x, y) \leq \frac{L}{2} \varphi(2 x, 2 y)
$$

for all $x, y \in X$. Suppose that $F: X \rightarrow\left(C_{c b}(Y), H\right)$ is a mapping satisfying

$$
H(F(x+y), F(x) \oplus F(y)) \leq \varphi(x, y)
$$

for all $x, y \in X$. Let $r$ and $M$ be positive real numbers with $r>1$ and $\operatorname{diam} F(x) \leq M\|x\|^{r}$ for all $x \in X$. Then there exists a unique set-valued additive mapping $A: X \rightarrow\left(C_{c b}(Y), H\right)$ such that

$$
H(F(x), A(x)) \leq \frac{L}{2-2 L} \varphi(x, x)
$$

for all $x \in X$. 
Proof. Let $y=x$ in (2.3). Since $F(x)$ is convex, we get

$$
H(F(2 x), 2 F(x)) \leq \varphi(x, x)
$$

and so

$$
H\left(F(x), 2 F\left(\frac{x}{2}\right)\right) \leq \varphi\left(\frac{x}{2}, \frac{x}{2}\right) \leq \frac{L}{2} \varphi(x, x)
$$

for all $x \in X$.

Consider

$$
S:=\left\{g: g: X \rightarrow C_{c b}(Y), g(0)=\{0\}\right\}
$$

and introduce the generalized metric on $X$,

$$
d(g, f)=\inf \{\mu \in(0, \infty): H(g(x), f(x)) \leq \mu \varphi(x, x), x \in X\},
$$

where, as usual, inf $\phi=+\infty$. It is easy to show that $(S, d)$ is complete (see [14, Theorem 2.4] and [25, Lemma 2.1]).

Now we consider the linear mapping $J: S \rightarrow S$ such that

$$
J g(x):=2 g\left(\frac{x}{2}\right)
$$

for all $x \in X$.

Let $g, f \in S$ be given such that $d(g, f)=\varepsilon$. Then

$$
H(g(x), f(x)) \leq \varepsilon \varphi(x, x)
$$

for all $x \in X$. Hence

$$
H(J g(x), J f(x))=H\left(2 g\left(\frac{x}{2}\right), 2 f\left(\frac{x}{2}\right)\right)=2 H\left(g\left(\frac{x}{2}\right), f\left(\frac{x}{2}\right)\right) \leq L \varepsilon \varphi(x, x)
$$

for all $x \in X$. So $d(g, f)=\varepsilon$ implies that $d(J g, J f) \leq L \varepsilon$. This means that

$$
d(J g, J f) \leq L d(g, f)
$$

for all $g, f \in S$.

It follows from (2.6) that $d(F, J F) \leq \frac{L}{2}$.

By Theorem 1.1, there exists a mapping $A: X \rightarrow Y$ satisfying the following:

(1) $A$ is a fixed point of $J$, i.e.,

$$
A\left(\frac{x}{2}\right)=\frac{1}{2} A(x)
$$

for all $x \in X$. The mapping $A$ is a unique fixed point of $J$ in the set

$$
M=\{g \in S: d(f, g)<\infty\} .
$$

This implies that $A$ is a unique mapping satisfying (2.7) such that there exists a $\mu \in(0, \infty)$ satisfying

for all $x \in X$;

$$
H(F(x), A(x)) \leq \mu \varphi(x, x)
$$

(2) $d\left(J^{n} F, A\right) \rightarrow 0$ as $n \rightarrow \infty$. This implies the equality

$$
\lim _{n \rightarrow \infty} 2^{n} F\left(\frac{x}{2^{n}}\right)=A(x)
$$

for all $x \in X$;

(3) $d(F, A) \leq \frac{1}{1-L} d(F, J F)$, which implies the inequality

$$
d(F, A) \leq \frac{L}{2-2 L} .
$$


This implies that the inequality (2.4) holds.

$$
\begin{aligned}
H(A(x+y), A(x) \oplus A(y)) & =\lim _{n \rightarrow \infty} 2^{n} H\left(F\left(\frac{x+y}{2^{n}}\right), F\left(\frac{x}{2^{n}}\right) \oplus F\left(\frac{y}{2^{n}}\right)\right) \\
& \leq \lim _{n \rightarrow \infty} 2^{n} \varphi\left(\frac{x}{2^{n}}, \frac{y}{2^{n}}\right)=0
\end{aligned}
$$

for all $x, y \in X$. Since $\operatorname{diam} F(x) \leq M\|x\|^{r}$ for all $x \in X$, diam $\left(2^{n} F\left(\frac{x}{2^{n}}\right)\right) \leq \frac{2^{n}}{2^{r n}} M\|x\|^{r}$ for all $x \in X$ and so $A(x)=2^{n} F\left(\frac{x}{2^{n}}\right)$ is a singleton set and $A(x+y)=A(x) \oplus A(y)$ for all $x, y \in X$.

Corollary 2.1. Let $p>1$ and $\theta \geq 0$ be real numbers, and let $X$ be a real normed space. Suppose that $F: X \rightarrow\left(C_{c b}(Y), H\right)$ is a mapping satisfying

$$
H(F(x+y), F(x) \oplus F(y)) \leq \theta\left(\|x\|^{p}+\|y\|^{p}\right)
$$

for all $x, y \in X$. Let $r$ and $M$ be positive real numbers with $r>1$ and $\operatorname{diam} F(x) \leq M\|x\|^{r}$ for all $x \in X$. Then there exists a unique set-valued additive mapping $A: X \rightarrow Y$ satisfying

$$
H(F(x), A(x)) \leq \frac{2 \theta}{2^{p}-2}\|x\|^{p}
$$

for all $x \in X$.

Proof. The proof follows from Theorem 2.2 by taking $L:=2^{1-p}$ and

$$
\varphi(x, y):=\theta\left(\|x\|^{p}+\|y\|^{p}\right)
$$

for all $x, y \in X$.

Theorem 2.3. Let $\varphi: X^{2} \rightarrow[0, \infty)$ be a function such that there exists an $L<1$ with

$$
\varphi(x, y) \leq 2 L \varphi\left(\frac{x}{2}, \frac{y}{2}\right)
$$

for all $x, y \in X$. Suppose that $F: X \rightarrow\left(C_{c b}(Y), H\right)$ is a mapping satisfying (2.3). Let $r$ and $M$ be positive real numbers with $r<1$ and $\operatorname{diam} F(x) \leq M\|x\|^{r}$ for all $x \in X$. Then there exists a unique set-valued additive mapping $A: X \rightarrow\left(C_{c b}(Y), H\right)$ such that

$$
H(F(x), A(x)) \leq \frac{1}{2-2 L} \varphi(x, x)
$$

for all $x \in X$.

Proof. It follows from (2.5) that

$$
H\left(F(x), \frac{1}{2} F(2 x)\right) \leq \frac{1}{2} \varphi(x, x)
$$

for all $x \in X$.

The rest of the proof is similar to the proof of Theorem 2.2.

Corollary 2.2. Let $1>p>0$ and $\theta \geq 0$ be real numbers, and let $X$ be a real normed space. Suppose that $F: X \rightarrow\left(C_{c b}(Y), H\right)$ is a mapping satisfying (2.8). Let $r$ and $M$ be positive real numbers with $r<1$ and $\operatorname{diam} F(x) \leq M\|x\|^{r}$ for all $x \in X$. Then there exists a unique set-valued additive mapping $A: X \rightarrow Y$ satisfying

$$
H(F(x), A(x)) \leq \frac{2 \theta}{2-2^{p}}\|x\|^{p}
$$

for all $x \in X$. 
Proof. The proof follows from Theorem 2.3 by taking $L:=2^{p-1}$ and

$$
\varphi(x, y):=\theta\left(\|x\|^{p}+\|y\|^{p}\right)
$$

for all $x, y \in X$.

\section{STABILITY OF THE SET-VALUED JENSEN ADDITIVE FUNCTIONAL EQUATION (1.2)}

Using the fixed point method, we prove the Hyers-Ulam stability of the set-valued Jensen additive functional equation (1.2).

Theorem 3.4. Let $\varphi: X^{2} \rightarrow[0, \infty)$ be a function such that there exists an $L<1$ with

$$
\varphi(x, y) \leq \frac{L}{2} \varphi(2 x, 2 y)
$$

for all $x, y \in X$. Suppose that $F: X \rightarrow\left(C_{c b}(Y), H\right)$ is a mapping satisfying $F(0)=\{0\}$ and

$$
H\left(2 F\left(\frac{x+y}{2}\right), F(x) \oplus F(y)\right) \leq \varphi(x, y)
$$

for all $x, y \in X$. Let $r$ and $M$ be positive real numbers with $r>1$ and $\operatorname{diam} F(x) \leq M\|x\|^{r}$ for all $x \in X$. Then there exists a unique set-valued Jensen additive mapping $A: X \rightarrow\left(C_{c b}(Y), H\right)$ such that

$$
H(F(x), A(x)) \leq \frac{1}{1-L} \varphi(x, 0)
$$

for all $x \in X$.

Proof. Let $y=0$ in (3.9). Since $F(x)$ is convex, we get

$$
H\left(F(x), 2 F\left(\frac{x}{2}\right)\right) \leq \varphi(x, 0)
$$

for all $x \in X$.

Consider

$$
S:=\left\{g: g: X \rightarrow C_{c b}(Y), g(0)=\{0\}\right\}
$$

and introduce the generalized metric on $X$,

$$
d(g, f)=\inf \{\mu \in(0, \infty): H(g(x), f(x)) \leq \mu \varphi(x, 0), x \in X\},
$$

where, as usual, inf $\phi=+\infty$. It is easy to show that $(S, d)$ is complete (see [14, Theorem 2.4] and [25, Lemma 2.1]).

Now we consider the linear mapping $J: S \rightarrow S$ such that

$$
J g(x):=2 g\left(\frac{x}{2}\right)
$$

for all $x \in X$.

It follows from (3.10) that $d(F, J F) \leq 1$.

The rest of the proof is similar to the proof of Theorem 2.2.

Corollary 3.3. Let $p>1$ and $\theta \geq 0$ be real numbers, and let $X$ be a real normed space. Suppose that $F: X \rightarrow\left(C_{c b}(Y), H\right)$ is a mapping satisfying $F(0)=\{0\}$ and

$$
H\left(2 F\left(\frac{x+y}{2}\right), F(x) \oplus F(y)\right) \leq \theta\left(\|x\|^{p}+\|y\|^{p}\right)
$$

for all $x, y \in X$. Let $r$ and $M$ be positive real numbers with $r>1$ and $\operatorname{diam} F(x) \leq M\|x\|^{r}$ for all $x \in X$. Then there exists a unique set-valued Jensen additive mapping $A: X \rightarrow Y$ satisfying

$$
H(F(x), A(x)) \leq \frac{2^{p} \theta}{2^{p}-2}\|x\|^{p}
$$


for all $x \in X$.

Proof. The proof follows from Theorem 3.4 by taking $L:=2^{1-p}$ and

$$
\varphi(x, y):=\theta\left(\|x\|^{p}+\|y\|^{p}\right)
$$

for all $x, y \in X$..

Theorem 3.5. Let $\varphi: X^{2} \rightarrow[0, \infty)$ be a function such that there exists an $L<1$ with

$$
\varphi(x, y) \leq 2 L \varphi\left(\frac{x}{2}, \frac{y}{2}\right)
$$

for all $x, y \in X$. Suppose that $F: X \rightarrow\left(C_{c b}(Y), H\right)$ is a mapping satisfying $F(0)=\{0\}$ and (3.9). Let $r$ and $M$ be positive real numbers with $r<1$ and $\operatorname{diam} F(x) \leq M\|x\|^{r}$ for all $x \in X$. Then there exists a unique set-valued Jensen additive mapping $A: X \rightarrow\left(C_{c b}(Y), H\right)$ such that

$$
H(F(x), A(x)) \leq \frac{L}{1-L} \varphi(x, 0)
$$

for all $x \in X$.

Proof. It follows from (3.11) that

$$
H\left(F(x), \frac{1}{2} F(2 x)\right) \leq \frac{1}{2} \varphi(2 x, 0) \leq L \varphi(x, 0)
$$

for all $x \in X$.

The rest of the proof is similar to the proofs of Theorems 2.2 and 3.4.

Corollary 3.4. Let $0<p<1$ and $\theta \geq 0$ be real numbers, and let $X$ be a real normed space. Suppose that $F: X \rightarrow\left(C_{c b}(Y), H\right)$ is a mapping satisfying $F(0)=\{0\}$ and (3.11). Let $r$ and $M$ be positive real numbers with $r<1$ and $\operatorname{diam} F(x) \leq M\|x\|^{r}$ for all $x \in X$. Then there exists a unique set-valued Jensen additive mapping $A: X \rightarrow Y$ satisfying

$$
H(F(x), A(x)) \leq \frac{2^{p} \theta}{2-2^{p}}\|x\|^{p}
$$

for all $x \in X$.

Proof. The proof follows from Theorem 3.5 by taking $L:=2^{p-1}$ and

$$
\varphi(x, y):=\theta\left(\|x\|^{p}+\|y\|^{p}\right)
$$

for all $x, y \in X$.

\section{REFERENCES}

[1] T. Aoki: On the stability of the linear transformation in Banach spaces. J. Math. Soc. Japan 2 (1950), $64-66$.

[2] K. J. Arrow and G. Debreu: Existence of an equilibrium for a competitive economy. Econometrica 22 (1954), $265-290$.

[3] J. P. Aubin and H. Frankowska: Set-Valued Analysis. Birkhäuser, Boston, 1990.

[4] R. J. Aumann: Integrals of set-valued functions. J. Math. Anal. Appl. 12 (1965), 1-12.

[5] T. Cardinali, K. Nikodem and F. Papalini: Some results on stability and characterization of K-convexity of set-valued functions. Ann. Polon. Math. 58 (1993), 185-192.

[6] T. Cascales and J. Rodrigeuz: Birkhoff integral for multi-valued functions. J. Math. Anal. Appl. 297 (2004), $540-560$.

[7] C. Castaing and M. Valadier: Convex Analysis and Measurable Multifunctions. Lect. Notes in Math. 580, Springer, Berlin, 1977.

[8] L. Cădariu and V. Radu: Fixed points and the stability of Jensen's functional equation. J. Inequal. Pure Appl. Math. 4, no. 1, Art. ID 4 (2003).

[9] L. Cădariu and V. Radu: On the stability of the Cauchy functional equation: a fixed point approach. Grazer Math. Ber. 346 (2004), 43-52.

[10] L. Cădariu and V. Radu: Fixed point methods for the generalized stability of functional equations in a single variable. Fixed Point Theory Appl. 2008, Art. ID 749392 (2008). 
[11] G. Debreu: Integration of correspondences. Proceedings of Fifth Berkeley Symposium on Mathematical Statistics and Probability, Vol. II, Part I (1966), 351-372.

[12] J. Diaz and B. Margolis: A fixed point theorem of the alternative for contractions on a generalized complete metric space. Bull. Am. Math. Soc. 74 (1968), 305-309.

[13] Iz. EL-Fassi: New stability results for the radical sextic functional equation related to quadratic mappings in (2, $\beta$ )-Banach spaces. J. Fixed Point Theory Appl. 20 (2018), no. 4, Art. 138, 17 pp.

[14] M. Eshaghi Gordji, C. Park and M. B. Savadkouhi: The stability of a quartic type functional equation with the fixed point alternative. Fixed Point Theory 11 (2010), 265-272.

[15] M. Eshaghi Gordji and M. B. Savadkouhi: Stability of a mixed type cubic-quartic functional equation in non-Archimedean spaces. Appl. Math. Letters 23 (2010), 1198-1202.

[16] P. Găvruta: A generalization of the Hyers-Ulam-Rassias stability of approximately additive mappings. J. Math. Anal. Appl. 184 (1994), 431-436.

[17] C. Hess: Set-valued integration and set-valued probability theory: an overview, in Handbook of Measure Theory. Vols. I, II, North-Holland, Amsterdam, 2002.

[18] W. Hindenbrand: Core and Equilibria of a Large Economy. Princeton Univ. Press, Princeton, 1974.

[19] D. H. Hyers: On the stability of the linear functional equation. Proc. Nat. Acad. Sci. USA 27 (1941), $222-224$.

[20] G. Isac and Th. M. Rassias: On the Hyers-Ulam stability of $\psi$-additive mappings. J. Approx. Theory 72 (1993), $131-137$.

[21] G. Isac and Th. M. Rassias: Stability of $\psi$-additive mappings: Applications to nonlinear analysis. Int. J. Math. Math. Sci. 19 (1996), 219-228.

[22] E. Klein and A. Thompson: Theory of Correspondence. Wiley, New York, 1984.

[23] K. Lee: Stability of functional equations related to set-valued functions (preprint).

[24] L. W. McKenzie: On the existence of general equilibrium for a competitive market. Econometrica 27 (1959), 54-71.

[25] D. Miheț and V. Radu: On the stability of the additive Cauchy functional equation in random normed spaces. J. Math. Anal. Appl. 343 (2008), 567-572.

[26] M. Mirzavaziri and M. S. Moslehian: A fixed point approach to stability of a quadratic equation. Bull. Braz. Math. Soc. 37 (2006), 361-376.

[27] K. Nikodem: On quadratic set-valued functions. Publ. Math. Debrecen 30 (1984), 297-301.

[28] K. Nikodem: On Jensen's functional equation for set-valued functions. Radovi Mat. 3 (1987), $23-33$.

[29] K. Nikodem: Set-valued solutions of the Pexider functional equation. Funkcialaj Ekvacioj 31 (1988), $227-231$.

[30] K. Nikodem: K-Convex and K-Concave Set-Valued Functions. Zeszyty Naukowe Nr. 559, Lodz, 1989.

[31] Y. J. Piao: The existence and uniqueness of additive selection for $(\alpha, \beta)-(\beta, \alpha)$ type subadditive set-valued maps. J. Northeast Normal University 41 (2009), 38-40.

[32] S. Pinelas, V. Govindan and K. Tamilvanan: Stability of a quartic functional equation. J. Fixed Point Theory Appl. 20 (2018), no. 4, Art. 148, 10 pp.

[33] D. Popa: Additive selections of $(\alpha, \beta)$-subadditive set-valued maps. Glas. Mat. Ser. III, 36 (56) (2001), 11-16.

[34] V. Radu: The fixed point alternative and the stability of functional equations. Fixed Point Theory 4 (2003), 91-96.

[35] Th. M. Rassias: On the stability of the linear mapping in Banach spaces. Proc. Am. Math. Soc. 72 (1978), $297-300$.

[36] L. Székelyhidi: Superstability of functional equations related to spherical functions. Open Math. 15 (2017), $427-432$.

[37] S. M. Ulam: Problems in Modern Mathematics. Chapter VI, Science ed., Wiley, New York, 1940.

HANYANG UNIVERSITY

RESEARCH INSTITUTE FOR NATURAL SCIENCES

SEOUL 04763, KOREA

Email address: baakehanyang.ac.kr

HANSHIN UNIVERSITY

DEPARTMENT OF FINANCIAL MATHEMATICS

GYEONGGI-DO 18101, KOREA

Email address: ssyun@hs.ac.kr

\section{DAEJIN UNIVERSITY}

DEPARTMENT OF MATHEMATICS

KYUNGGI 11159, KOREA

Email address: jrleeddaejin.ac.kr 
UNIVERSITY OF SEOUL

DEPARTMENT OF MATHEMATICS

SEOUL 02504, KOREA

Email address: dyshineuos.ac.kr 